\title{
ARTÍCULOS
}

\section{Producción y descomposición de hojarasca en la selva ombrófila mixta en el sur de Brasil}

\author{
Litter production and decomposition in a mixed araucaria forest in the south of Brazil
}

\author{
Silvana L Caldato**, Paulo A Floss ${ }^{\mathrm{b}}$, Elzira C Serafini Floss ${ }^{\mathrm{c}}$ \\ *Autor de Correspondencia: ${ }^{a}$ Universidade Federal de Santa Maria, Departamento de Ciências Florestais, Campus Universitário, \\ UFSM, Camobi, 97105-900, Santa Maria, RS, Brasil, scaldato@yahoo.com.br \\ bUniversidade Federal de Santa Maria, EPAGRI/Centro de Pesquisas de Pequenas Propriedades, Chapecó, SC, Brasil. \\ ${ }^{c}$ Universidade Federal de Santa Maria, Centro de Ciências Naturais e Exatas, Brasil.
}

\begin{abstract}
SUMMARY
The aim of the present work was to evaluate the litter production and decomposition in a mixed araucaria forest in the south of Brazil. The vegetation of the area is characterized by two forest types: forest type I, with the upper canopy dominated by Araucaria angustifolia; and forest type II, with higher diversity of tree species, predominantly broad-leafed species in the dominant canopy strata. To evaluate the litter production, 20 wood traps with $0.25 \mathrm{~m}^{2}$ of surface were installed. Ten samples of $0.25 \mathrm{~m}^{2}$ were monthly collected from the litter layer. Litter decomposition was evaluated from the instantaneous decay rate (K). The average of three years concluded that litter production did not differ significantly in the two types of forest studied: 7,661 kg ha-1 year-1 in forest type I and 8,624 $\mathrm{kg} \mathrm{ha}^{-1}$ year ${ }^{-1}$ in forest type II. The months with the highest production in forest type II were correlated with periods of higher precipitation (spring and summer). The litter layer was significantly different for the two forest types, being heavier in forest type I with values of $9,924 \mathrm{~kg} \mathrm{ha}^{-1}$ and $7,906 \mathrm{~kg} \mathrm{ha}^{-1}$ for forest type II. The area with predominance of Araucaria angustifolia presented a slower rate of litter decomposition and nutrient release.
\end{abstract}

Key words: litter production, decay rate, Araucaria angustifolia.

\section{RESUMEN}

El objetivo del presente trabajo fue evaluar la producción y descomposición de hojarasca de la selva ombrófila mixta en el sur de Brasil. La vegetación del área presentó dos tipos forestales: I, cuyo dosel superior estaba dominado por Araucaria angustifolia y II, con mayor diversidad de especies arbóreas predominando latifoliadas en el estrato superior. Para evaluar la producción de hojarasca fueron instalados 20 colectores de $0,25 \mathrm{~m}^{2}$ de superficie. Para la cuantificación del mantillo, mensualmente se retiraron 10 muestras de $0,25 \mathrm{~m}^{2}$. La descomposición de la hojarasca fue determinada a partir de la tasa instantánea de descomposición (K). De los resultados observados en tres años de estudio se concluye que la producción de hojarasca no difirió significativamente en los dos tipos forestales estudiados, con $7.661 \mathrm{~kg} \mathrm{ha}^{-1} \mathrm{año}^{-1}$ en el tipo forestal I y $8.624 \mathrm{~kg} \mathrm{ha}^{-1} \mathrm{año}^{-1}$ en el tipo forestal II. Los meses de mayor cantidad de hojarasca en el tipo forestal II estuvieron correlacionados con períodos de mayores precipitaciones (primavera y verano). Para el mantillo hubo diferencias significativas entre los dos tipos forestales, presentando mayor cantidad el tipo forestal I con valores de $9.924 \mathrm{~kg} \mathrm{ha}^{-1}$ y de $7.906 \mathrm{~kg} \mathrm{ha}^{-1}$ el tipo forestal II. El bosque con predominio de Araucaria angustifolia presentó una tasa de descomposición y una liberación de nutrientes más lentas.

Palabras clave: producción hojarasca, tasa de descomposición, Araucaria angustifolia.

\section{INTRODUCCIÓN}

La selva ombrófila mixta, también conocida como bosque de araucaria o pino-brasilero (Rambo 1956, Klein 1960, Hueck 1972), caracterizada por la presencia de elementos Coniferales y Laurales (Leite y Klein 1990), es un ecosistema que siempre identificó la región sur de Brasil por ser su área de mayor distribución y principalmente por la fisonomía de su especie dominante, la Araucaria angustifolia (Bert.) Kuntze. Su distribución en Brasil, según Klein (1960) y Hueck (1972), ocurre en los estados de Rio Grande do Sul, Santa Catarina, Paraná y sur de São Paulo, con manchas aisladas en los estados de Rio de Janeiro y Minas Gerais; también con presencia en la provincia de Misiones en Argentina y en el oeste de Paraguay. Debido a la acción antropogénica con la deforestación y el uso intensivo de los suelos, permanece actualmente sólo el $2 \%$ de la extensa área original de este ecosistema (Gubert 
Filho 1990). En este sentido, las áreas de reservas de diferentes ecosistemas cumplen un papel fundamental en la conservación de la biodiversidad.

El funcionamiento de los ecosistemas forestales depende en gran medida de la descomposición del manto orgánico de restos vegetales sobre el suelo, el cual tiene un papel clave en el reciclaje de nutrientes (Polyakova y Billor 2007). El manto orgánico de hojas, ramas, frutos, corteza, etc., que se forma sobre el suelo de un bosque es llamado mantillo. La descomposición del material orgánico que cae de los árboles hasta el suelo es una de las principales fuentes de carbono orgánico y de nutrientes (principalmente nitrógeno y fósforo) y da origen al "ciclo de nutrientes" dentro del sistema suelo-planta (Olson 1963, Attiwill 1968, O’Connell 1988, Foelkel 2008).

La tasa de descomposición del mantillo puede ser determinada por la relación entre la cantidad que cae anualmente con la acumulada en el suelo. De acuerdo con Poggiani y Schumacher (2004) y Pallardy (2008) la forma y velocidad de descomposición están relacionadas principalmente con las condiciones climáticas y fundamentalmente con la relación $\mathrm{C} / \mathrm{N}$, afín con los microorganismos que efectúan el proceso. Normalmente, las hojas verdes presentan mayor tasa de descomposición que las hojas senescentes en razón de su concentración inicial más elevada de nitrógeno y de fósforo, y los materiales más lignificados son más resistentes a la descomposición (Reis y Barros 1990). La tasa de mineralización del nitrógeno en el piso del bosque está correlacionada con la mineralización total del nitrógeno (mantillo y suelo) y depende además de la calidad de la hojarasca (expresada como las tasas $\mathrm{C} / \mathrm{N}$ o lignina/nitrógeno) (Vogt et al. 1986, Stump y Binkley 1993).

Varias investigaciones han sido desarrolladas en el sentido de entender la dinámica del mantillo, su acumulación, descomposición y calidad (Cunha et al. 1993, Figueiredo Filho et al. 2003, Schumacher et al. 2004, Vogel et al. 2007, Wang et al. 2008) y el ciclo de sus nutrientes (Caldeira 2003, Poggiani y Schumacher 2004, Polyakova y Billor 2007).

El objetivo del presente trabajo fue cuantificar la producción de hojarasca y la descomposición del mantillo en dos tipos forestales de la selva ombrófila mixta en la región de Caçador en el estado de Santa Catarina, Brasil.

\section{MÉTODOS}

Caracterización del área. El área de selva ombrófila mixta estudiada posee aproximadamente 772 ha, se localiza en la Reserva Forestal de Caçador en Santa Catarina, Brasil, perteneciente a EMBRAPA (Empresa Brasilera de Pesquisa Agropecuaria) y está cedida en régimen de comodato a EPAGRI (Empresa de Pesquisa Agropecuaria e Extensão Rural de Santa Catarina). Localizada en las coordenadas geográficas $26^{\circ} 50^{\prime}-26^{\circ} 54^{\prime} \mathrm{S}$ y $51^{\circ} 00^{\prime}-50^{\circ} 55^{\prime} \mathrm{O}$, en una altitud media de $1.100 \mathrm{~m}$ s.n.m. De acuerdo con Nimer (1990) el clima de la región según la clasificación climática de Köppen es el Cfb, templado húmedo con heladas severas. La temperatura media anual es de $16^{\circ} \mathrm{C}$, con media de la temperatura del mes más cálido inferior a $21^{\circ} \mathrm{C}$ y en los meses más fríos inferior a $10^{\circ} \mathrm{C}$. La precipitación media anual es de $1.800 \mathrm{~mm}$, con distribución normal durante todo el año (figura 1).

Los suelos de la región son rojos, profundos, comprendiendo según Moser (1990) suelos minerales, no hidromórficos, con horizonte B de arcilla de actividad baja, de fertilidad variable y baja disponibilidad de fósforo. Ocurren normalmente en relieve ondulado y fuertemente ondulado, con vegetación de sabana y selva ombrófila mixta.

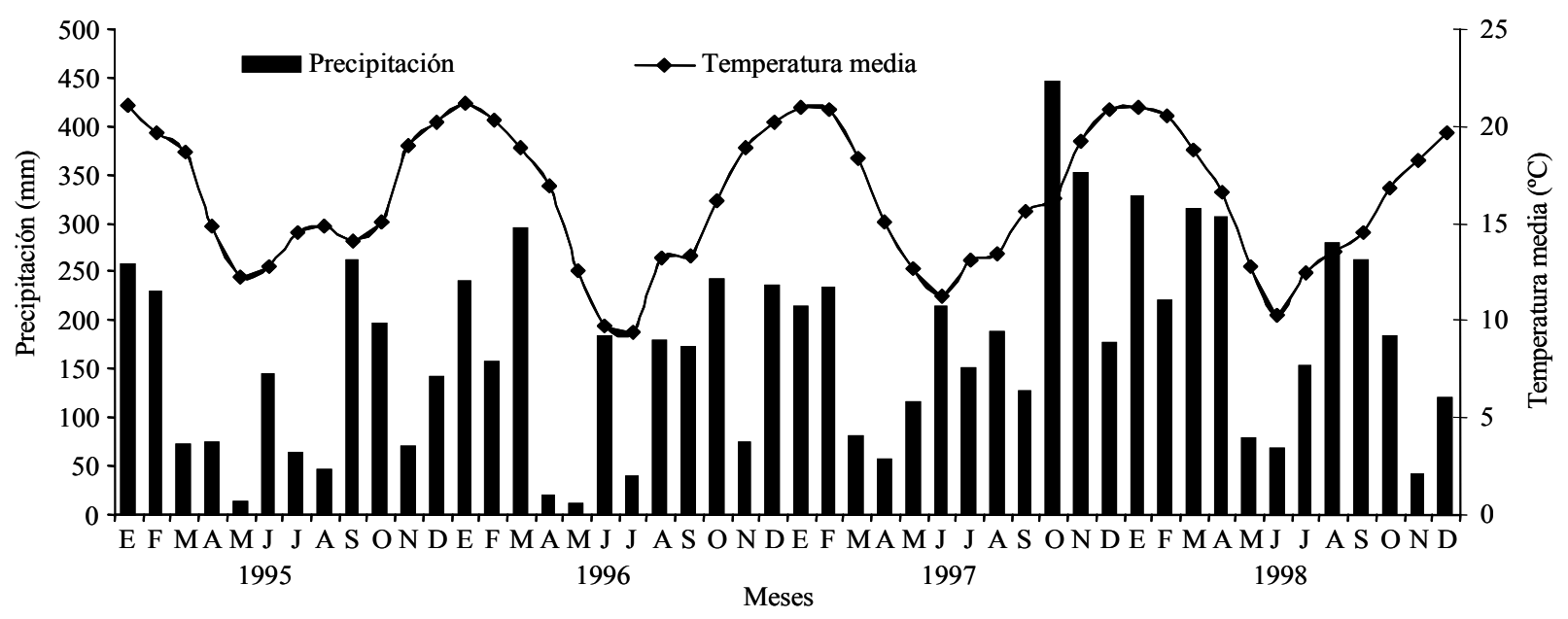

Figura 1. Precipitación y temperatura de enero de 1995 a diciembre de 1998 en la Reserva Forestal de Caçador, Santa Catarina, Brasil.

Rainfall and temperature from January 1995 to December 1998 in Forest Reserve of Caçador in Santa Catarina, Brazil. 
La vegetación de la región presenta a A. angustifolia como la especie dominante en el estrato superior del bosque, el que está acompañado por diversos tipos de sotobosques (Klein 1978). En el área del estudio el subdosel está compuesto principalmente por Ocotea porosa (Nees) Barroso, Ilex paraguariensis A. St. Hil., además de Cedrela fissilis Vell., Prunus sellowii Koehne, Ocotea pulchella (Nees) Mez, Nectandra megapotamica (Spreng.) Mez, Ocotea pretiosa (Nees) Benth. et Hook., Cupania vernalis Camb., Piptocarpha angustifolia Dusén ex Malme, Ilex brevipuspis Reissek, entre otras.

DaCroce (1991), en un estudio fitosociológico y de caracterización espacial y estructural de la presente Reserva, clasificó el bosque en dos tipos forestales principales: tipo forestal I, con predominancia de A. angustifolia en el estrato superior; tipo forestal II, donde la diversidad de especies es mayor, con supremacía de otras especies de latifoliadas en el estrato superior.

Producción de hojarasca. Para estimar la cantidad de hojarasca producida fueron instalados 20 colectores de madera con área de $0,25 \mathrm{~m}^{2}, 10 \mathrm{~cm}$ de altura y fondo con tela de nailon con malla de $2 \times 2 \mathrm{~mm}$, situados a $20 \mathrm{~cm}$ arriba del suelo. Los colectores fueron distribuidos en parcelas permanentes de 25 x $10 \mathrm{~m}$ (utilizadas por DaCroce 1991 para estudio fitosociológico), utilizando dos colectores por parcela (cinco parcelas en el tipo forestal I y cinco en el tipo forestal II). Para cuantificar la hojarasca acumulada sobre el suelo (mantillo), en las mismas parcelas utilizadas para cuantificar la producción de hojarasca fue retirada mensualmente una muestra al azar con dimensiones de $0,25 \mathrm{~m}^{2}$, totalizando 10 muestras mensuales, utilizando un cuadro de madera y un cuchillo para demarcar el área a ser colectada. La hojarasca depositada en los colectores y el mantillo fueron recolectados en el inicio de cada mes, durante el período de septiembre de 1995 a agosto de 1998 , totalizando 36 recolecciones. Todo el material colectado fue llevado al laboratorio de la EPAGRI para secamiento en estufa a $60^{\circ} \mathrm{C}$, hasta peso constante.

Los valores de hojarasca producida y mantillo en los dos tipos forestales fueron comparados por la prueba de Tukey $(P<0,05)$. La verificación de la influencia de las variables temperatura y precipitación sobre la producción de hojarasca y el mantillo fue realizada usando el coeficiente de correlación lineal de Pearson.

Descomposición del mantillo. La descomposición del mantillo fue analizada por medio de la tasa instantánea de descomposición $(\mathrm{K})$, que consiste en la relación entre la biomasa total producida (L) dividida por la cantidad media de mantillo acumulado (Xss). Los períodos de tiempo, en años, necesarios para la descomposición de 50\% y $95 \%$ de una determinada porción de mantillo fueron calculados por las respectivas ecuaciones [1] y [2] de acuerdo con Olson (1963):

$$
\begin{gathered}
t_{0,5}=-\ln 0,5 K^{-1} \\
t_{0,05}=3 K^{-1}
\end{gathered}
$$

\section{RESULTADOS}

La producción de hojarasca para los dos tipos forestales durante los tres años de estudio no se diferenció significativamente $(P>0,05)$; los valores fueron de $7.661 \mathrm{~kg} \mathrm{ha}^{-1}$ año $0^{-1}$ en el tipo forestal I (desviación estándar de 1.913 $\mathrm{kg} \mathrm{ha}^{-1} \mathrm{año}^{-1}$ ) y $8.624 \mathrm{~kg} \mathrm{ha}^{-1} \mathrm{año}^{-1}$ en el tipo forestal II (desviación estándar de $1.567 \mathrm{~kg} \mathrm{ha}^{-1} \mathrm{año}^{-1}$ ). La figura 2A presenta la distribución mensual de la producción de hojarasca (promedio de tres años) y la distribución de la precipitación.

El mantillo (promedio de 3 años) difirió significativamente por la prueba de Tukey $(P<0,05)$ para los dos tipos forestales estudiados. Los valores obtenidos fueron de $9.924 \mathrm{~kg} \mathrm{ha}^{-1}$ para el tipo forestal I (desviación estándar
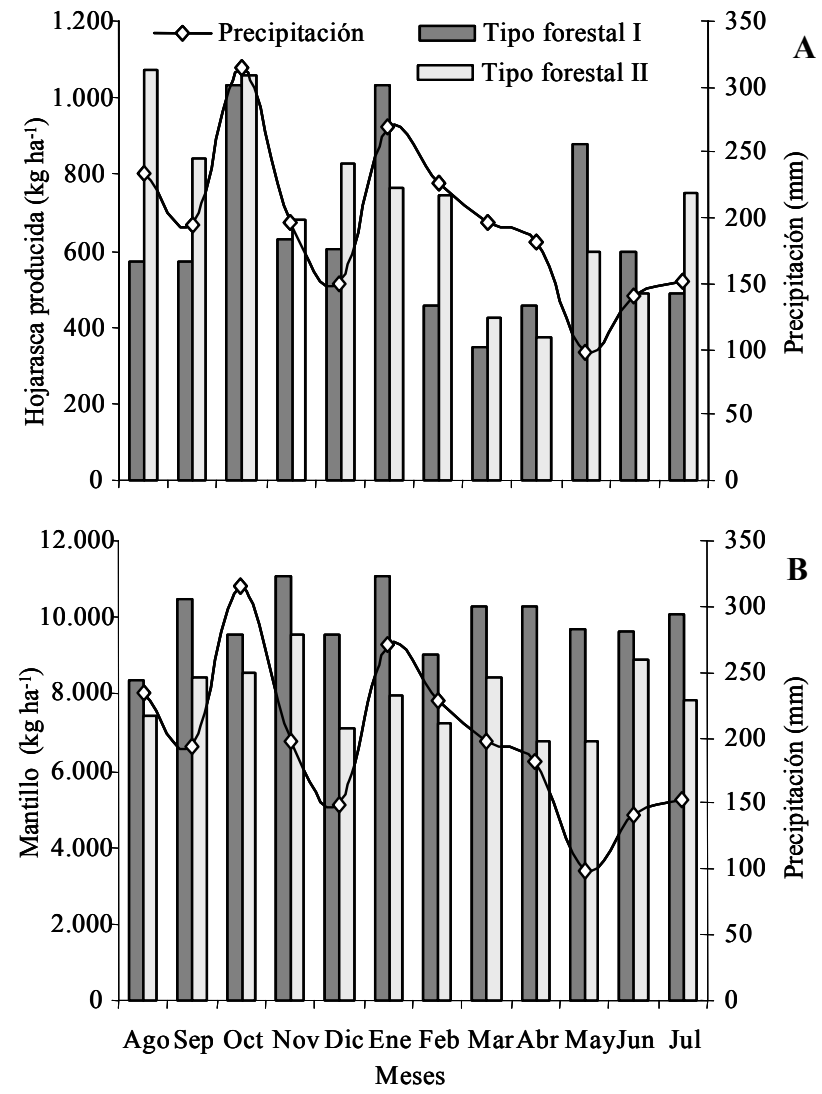

Figura 2. (A) Producción mensual de hojarasca y (B) mantillo (promedio de tres años) en los tipos forestales I y II, y precipitación mensual en la Reserva Forestal de Caçador, en Santa Catarina, Brasil.

(A) Monthly litter production and (B) litter layer weight variation (average of three years) in forest types I and II, and monthly precipitation in the Forest Reserve of Caçador, in Santa Catarina, Brazil. 
de $1.564 \mathrm{~kg} \mathrm{ha}^{-1}$ ) y de $7.906 \mathrm{~kg} \mathrm{ha}^{-1}$ para el tipo forestal II (desviación estándar de $1.813 \mathrm{~kg} \mathrm{ha}^{-1}$ ). La figura 2B presenta el mantillo acumulado en el suelo y la distribución de la precipitación.

En la matriz de coeficiente de correlación (cuadro 1) se puede observar que la producción de hojarasca en el tipo forestal II fue la única variable que tuvo correlación significativa $(\mathrm{r}=0,55 ; P<0,01)$ con la precipitación.

Los valores obtenidos para la constante de descomposición instantánea $(\mathrm{K})$ fueron de 0,77 y 1,09 para los tipos forestales I y II, respectivamente. El tiempo medio estimado para que ocurra la descomposición del 50\% del mantillo fue de 0,90 años para el tipo forestal I y de 0,64 años para el tipo forestal II. La descomposición del 95\% del material necesitó de un tiempo medio estimado de 3,89 años para el tipo forestal I y de 2,75 años para el tipo forestal II.

Cuadro 1. Valores de coeficiente de correlación entre las variables precipitación ( $\mathrm{PP})$, temperatura $\left(\mathrm{T}^{\circ}\right)$, mantillo en el tipo forestal I (Ac I), mantillo en el tipo forestal II (Ac II), hojarasca producida en el tipo forestal I (Prod I) y hojarasca producida en el tipo forestal II (Prod II) en la Reserva Forestal de Caçador, en Santa Catarina, Brasil.

Correlation coefficient values among the variables precipitation (PP), temperature $\left(\mathrm{T}^{\circ}\right)$, litter accumulated forest type I (Ac I), litter accumulated forest type II (Ac II), litter produced type forest I (Prod I) and litter produced type forest II (Prod II) in the Forest Reserve of Caçador, in Santa Catarina, Brazil.

\begin{tabular}{lrrrrrr}
\hline & PP & $\mathrm{T}^{\mathbf{o}}$ & Ac I & Ac II & Prod I & Prod II \\
\hline PP & 1 & 0,462 & 0,004 & 0,276 & 0,391 & $0,554^{* *}$ \\
$\mathrm{~T}^{\circ}$ & & 1 & 0,282 & $-0,021$ & 0,070 & 0,086 \\
Ac I & & & 1 & 0,444 & 0,168 & $-0,399$ \\
Ac II & & & & 1 & 0,069 & 0,047 \\
Prod I & & & & & 1 & 0,436 \\
Prod II & & & & & & 1 \\
\hline
\end{tabular}

** $P<0,01$

\section{DISCUSIÓN}

Los valores de producción de hojarasca observados para los tipos forestales I y II de la selva ombrófila mixta son semejantes a los resultados verificados por otros autores para este tipo de selva en diferentes regiones. Por ejemplo, Figueiredo Filho et al. (2003) observaron en Paraná valores de $7.737 \mathrm{~kg} \mathrm{ha}^{-1} \mathrm{año}^{-1}$, siendo la primavera la estación que presentó la mayor cantidad de hojarasca, seguida por el invierno, verano y otoño; respondiendo a los meses de mayor precipitación. Vogel et al. (2007) encontraron valores para la devolución media anual de $7.436 \mathrm{~kg} \mathrm{ha}^{-1}$ en el bosque estacional decidual en Rio Grande do Sul y concluyen que durante los dos años de estudio la mayor devolución de hojarasca fue en el inicio del invierno y principalmente en la primavera. También en Rio Grande do Sul, Schumacher et al. (2004), estudiando una plantación de A. angustifolia con 17 años de edad, observaron una producción total anual de hojarasca que alcanzó $6.960 \mathrm{~kg} \mathrm{ha}^{-1}$. Para Misiones, en Argentina, Pérez et al. (2006) en plantaciones de 20 años de edad de Pinus taeda L. encontraron una caída de acículas de $9.520 \mathrm{~kg} \mathrm{ha}^{-1} \mathrm{año}^{-1}$. En Australia Bubb et al. (1998) verificaron para Araucaria cunninghamii Sweet que la mayor producción de hojarasca ocurrió en primavera, con producción anual de 6.000 y $10.900 \mathrm{~kg} \mathrm{ha}^{-1}$ para los rodales jóvenes (10 y 14 años) y poblaciones maduras de 62 años de edad, respectivamente.

Los valores del mantillo acumulado en el suelo concuerdan con los encontrados por Caldeira (2003) en la selva ombrófila mixta montana en Paraná, reportando $7.900 \mathrm{~kg}$ $\mathrm{ha}^{-1}$ (para el estadio inicial), $8.600 \mathrm{~kg} \mathrm{ha}^{-1}$ (para el estadio intermedio) y $7.600 \mathrm{~kg} \mathrm{ha}^{-1}$ (para el estadio avanzado). Para la selva estacional decidual en Rio Grande do Sul, Cunha et al. (1993) registraron una cantidad media de mantillo sobre el suelo de $6.700 \mathrm{~kg} \mathrm{ha}^{-1}$. En Europa, Novak y Slodicak (2008), estudiando la cantidad y calidad de hojarasca en bosques jóvenes dominados por latifoliadas con Fagus sylvatica L., obtuvieron valores anuales que variaron de 2.200 a $5.300 \mathrm{~kg} \mathrm{ha}^{-1}$.

De acuerdo con Poggiani y Schumacher (2004) el clima y la geografía son los aspectos más importantes en el ciclo de nutrientes de bosques naturales, resaltando que la cantidad de hojarasca que se acumula en el suelo es dependiente principalmente de la composición de las especies, de la intensidad de la cobertura forestal, de la edad y del sitio. Para el bosque estudiado, se puede observar una estacionalidad con menores valores de producción al inicio del otoño, en los dos tipos forestales, y las mayores producciones con máximos principalmente en primavera y verano, período en que ocurren las mayores precipitaciones pluviométricas. Backes et al. (2005), en estudio de la selva ombrófila mixta de São Francisco de Paula en Rio Grande do Sul, también verificaron un aumento significativo en la caída de hojas y acículas durante la primavera y la menor producción en el invierno, y atribuyeron este patrón a la precipitación, infiriendo que el aumento significativo de la caída durante la primavera y verano puede estar relacionado con el aumento de la precipitación durante este período.

Los resultados obtenidos de la tasa de descomposición del mantillo indican que en el tipo forestal I la descomposición es más lenta y en el tipo forestal II es más rápida. De acuerdo con el criterio adoptado, los bosques con un valor de $\mathrm{K}$ superior a 1 producen hojarasca de rápida descomposición (Olson 1963). Dentro de una misma región climática, el principal factor en la descomposición de la hojarasca es la calidad del sustrato. En este sentido, la tasa de descomposición de las acículas de A. angustifolia que domina el dosel superior en el tipo forestal I es menor que 
la de las de especies latifoliadas (dominantes en el tipo forestal II). También Wang et al. (2008) concluyen que la tasa de descomposición de la hojarasca de la conífera Cunninghamia lanceolata (Lamb.) Hook. es más lenta que la de la latifoliada Michelia macclurei Dandy, y que no difiere significativamente entre plantíos puros y mixtos. Sin embargo, es levemente mayor en plantíos mixtos. En Australia, Bubb et al. (1998) verificaron una tasa de descomposición del follaje de A. cunninghamii relativamente baja para un bosque subtropical, con un tiempo estimado para descomponer 50\% del material de 1,7 años. Según los autores, la descomposición del mantillo estuvo fuertemente correlacionada con los indicadores de calidad de la hojarasca como contenidos de nitrógeno, carbono, fósforo, lignina y polifenoles, y las relaciones carbono/nitrógeno y lignina/ nitrógeno. Igualmente, Ribeiro et al. (2007) mencionan una tasa de descomposición de acículas de Pinus pinaster Ait. inferior a la determinada para hojas de Eucalyptus globulus Labill., siendo la diferencia más acentuada en la fase inicial de descomposición. Los autores atribuyen las diferencias principalmente a los elevados contenidos de lignina en las acículas de $P$. pinaster. En general, las hojas de especies de maderas duras (latifoliadas) se descomponen de manera más rápida que las hojas de coníferas, comúnmente también las hojas y el mantillo de latifoliadas contienen mayores concentraciones de nitrógeno que las hojas de coníferas (Pallardy 2008). De este modo, de acuerdo con Berg (2000) y Wang et al. (2007), la tasa de descomposición de la hojarasca está positivamente correlacionada con la concentración inicial de nitrógeno.

\section{CONCLUSIONES}

La producción de hojarasca no difiere significativamente en los dos tipos forestales estudiados y los meses con mayor cantidad en el tipo forestal II (predominio de latifoliadas) están correlacionados positivamente con períodos de mayores precipitaciones.

El mantillo presenta mayor acumulación en el tipo forestal I (predominio de A. angustifolia), que en el tipo forestal II. Esto permite inferir una tasa de descomposición más lenta en el mantillo del tipo forestal I. El menor tiempo de descomposición en el tipo forestal II, donde la diversidad de especies es mayor, indica que los nutrientes se tornan disponibles en un período menor. Sin embargo, en ambos ecosistemas la descomposición es bastante rápida.

\section{AGRADECIMIENTOS}

A la Empresa de Pesquisa Agropecuaria e Extensão Rural de Santa Catarina (EPAGRI) por la concesión del área de estudio y el apoyo logístico.

\section{REFERENCIAS}

Attiwill PM. 1968. The loss of nutrients from decomposing litter. Ecology 49: 142-145.

Backes A, FL Prates, MG Viola. 2005. Produção de serapilheira em floresta ombrófila mista, em São Francisco de Paula, Rio Grande do Sul, Brasil. Acta Botânica Brasilica 19: 155-160.

Berg B. 2000. Litter decomposition and organic matter turnover in northern forest soils. Forest Ecology and Management 133: 13-22.

Bubb KA, H Xu, JA Simpson, PG. Saffigna. 1998. Some nutrient dynamics associated with litterfall and litter decomposition in hoop pine plantations of southeast Queensland, Australia. Forest Ecology and Management 110: 343-352.

Caldeira MVW. 2003. Determinação de biomassa e nutrientes em uma floresta ombrófila mista Montana em General Carneiro, Paraná. Tesis Doctor Ciências Florestais. Curitiba, Brasil. Setor de Ciências Agrárias, Universidade Federal do Paraná. 176 p.

Cunha GC, LA Grendene, MA Durlo, DA Bressan. 1993. Dinâmica nutricional em floresta estacional decidual com ênfase aos minerais provenientes da deposição da serapilheira. Ciência Florestal 3: 35-40.

DaCroce DM. 1991. Caracterização especial estrutural e fitossociológica da Reserva Genética Florestal de Caçador SC, através da análise de componentes principais e sistemas de informações geográficas. Tesis Magíster. Santa Maria, Brasil. Centro de Ciências Rurais, Universidade Federal de Santa Maria. 120 p.

Figueiredo Filho A, G Ferreira Moraes, L Budant Schaaf, DJ Figueiredo. 2003. Avaliação estacional da deposição de serapilheira em uma floresta ombrófila mista localizada no sul do estado do Paraná. Ciência Florestal 13: 11-18.

Foelkel C. 2008. Minerais e nutrientes das árvores dos eucaliptos: Aspectos ambientais, fisiológicos, silviculturais e industriais acerca dos elementos inorgânicos presentes nas árvores. Eucalyptus on line book and newsletter. Consultado 18 nov. 2008. Disponible en http://www.eucalyptus.com.br/

Gubert Filho F. 1990. Proposta para a criação de um sistema de unidades de conservação da Araucaria angustifolia no Estado do Paraná. Silvicultura 42: 287-300.

Hueck K. 1972. As florestas da América do sul. São Paulo, Brasil. Polígono. 466 p.

Klein RM. 1960. O aspecto dinâmico do pinheiro-brasileiro. Sellowia 12: 17-48.

Klein RM. 1978. Mapa fitogeográfico do estado de Santa Catarina. Itajaí, Brasil. SUDESUL/FATMA/HBR. 24 p.

Leite PF, RM Klein. 1990. Vegetação. In Instituto Brasileiro de Geografia e Estatística ed. Geografia do Brasil: Região Sul. Rio de Janeiro, Brasil. p. 113-150.

Moser JM. 1990. Solos. In Instituto Brasileiro de Geografia e Estatística ed. Geografia do Brasil: Região Sul. Rio de Janeiro, Brasil. p. 95-111.

Nimer E. 1990. Clima. In Instituto Brasileiro de Geografia e Estatística ed. Geografia do Brasil: Região Sul. Rio de Janeiro, Brasil. p. 151-187.

Novak J, M Slodicak. 2008. Quantity and quality of litter-fall in young European beech (Fagus sylvatica L.) stands in localities naturally dominated broadleaves. Austrian Journal of Forest Science 125: 67-78. 
O'Connell AM. 1988. Nutrient dynamics in decomposing litter in karri (Eucalyptus diversicolor F Muell) forests of southwestern Australia. Journal of Ecology. 76: 1186-1203.

Olson JS. 1963. Energy storage and the balance of decomposers in ecological systems. Ecology 44: 322-31.

Pallardy SG. 2008. Physiology of woody plants. $3^{\text {rd }}$ ed. New York, USA. Elsevier. 454 p.

Pérez CA, JF Goya, F Bianchini, JL Frangi, R Fernández. 2006. Productividad aérea y ciclo de nutrientes en plantaciones de Pinus taeda L. en el norte de la provincia de Misiones, Argentina. Interciencia 31: 794-801.

Poggiani F, MV Schumacher. 2004. Nutrient cycling in native forests. In Gonçalves JLM, V Benedetti eds. Forest nutrition and fertilization. Piracicaba, Brasil. IPEF. p. 287-305.

Polyakova O, N Billor. 2007. Impact of deciduous tree species on litterfall quality, decomposition rates and nutrient circulation in pine stands. Forest Ecology and Management 253: 11-18.

Rambo B. 1956. A fisionomia do Rio Grande do Sul. Porto Alegre, Brasil. Selbach. 456 p.

Ribeiro C, N Cortez, A Martins, A Azevedo, M Madeira. 2007. Decomposição de agulhas de Pinus pinaster e de folhas de Eucalyptus globulus em regiões do interior e do litoral de Portugal. Rev. de Ciências Agrárias 30: 142-158.

Reis MGF, NF Barros. 1990. Ciclagem de nutrientes em plantios de eucalipto. In Barros NF, RF Novais eds. Relação solo-eucalipto. Viçosa, Brasil. Editora Folha de Viçosa. p. 265-302.

Schumacher MV, EJ Brun, JI Hernandes, FG König. 2004. Produção de serapilheira em uma floresta de Araucaria angustifolia (Bertol.) Kuntze no município de Pinhal Grande-RS. Revista Árbore 28: 29-37.

Stump LM, D Binkley. 1993. Relationships between litter quality and nitrogen availability in Rocky Mountain forests. Can. J. For. Res. 23: 492-502.

Vogel HLM, MV Schumacher, P Trüby, E Vuaden. 2007. Avaliação da devolução de serapilheira em uma floresta estacional decidual em Itaara, RS, Brasil. Ciência Florestal 1: 187-196.

Vogt, KA, CC Grier, DJ Vogt. 1986. Production, turnover and nutrient dynamics of above and belowground detritus of world forests. Advances Ecol. Res. 15: 303-372.

Wang Q, S Wang, B Fan, X Yu. 2007. Litter production, leaf litter decomposition and nutrient return in Cunninghamia lanceolata plantations in south China: effect of planting conifers with broadleaved species. Plant Soil 297: 201-211.

Wang Q, S Wang, Y Huang. 2008. Comparisons of litterfall, litter decomposition and nutrient return in a monoculture Cunninghamia lanceolata and a mixed stand in southern China. Forest Ecology and Management 255: 1210-1218.

Recibido: 07.05.09

Aceptado: 05.10.09 